Mots. Les langages du politique

$117 \mid 2018$

Les «petites phrases »

Catherine Kerbrat-Orecchioni, Les débats de l'entredeux-tours des élections présidentielles françaises. Constantes et évolutions d'un genre

Paris, L'Harmattan, 2017, 372 p.

Paul Bacot

\title{
OpenEdition
}

Journals

Édition électronique

URL : https://journals.openedition.org/mots/23516

DOI : $10.4000 /$ mots. 23516

ISSN : 1960-6001

Éditeur

ENS Éditions

Édition imprimée

Date de publication : 5 juillet 2018

Pagination : 149-152

ISSN : 0243-6450

Référence électronique

Paul Bacot, "Catherine Kerbrat-Orecchioni, Les débats de l'entre-deux-tours des élections présidentielles françaises. Constantes et évolutions d'un genre », Mots. Les langages du politique [En ligne], 117| 2018, mis en ligne le 05 juillet 2018, consulté le 23 avril 2022. URL : http://journals.openedition.org/mots/ 23516 ; DOI : https://doi.org/10.4000/mots.23516

Ce document a été généré automatiquement le 23 avril 2022

(c) ENS Éditions 


\section{Catherine Kerbrat-Orecchioni, Les débats de l'entre-deux-tours des élections présidentielles françaises. Constantes et évolutions d'un genre}

Paris, L'Harmattan, 2017, 372 p.

\section{Paul Bacot}

\section{RÉFÉRENCE}

Catherine Kerbrat-Orecchioni, Les débats de l'entre-deux-tours des élections présidentielles françaises. Constantes et évolutions d'un genre, Paris, L'Harmattan, 2017, 372 p.

1 C'est le débat par excellence : celui qui oppose entre les deux tours de chaque élection présidentielle française les deux candidats arrivés en tête au premier et qui se trouvent donc qualifiés pour le second, devenant les finalistes de la compétition - puisque ce vocabulaire sportif s'est progressivement imposé. Ce rituel qui bat régulièrement des records d'audience télévisée méritait à coup sûr à lui seul cette étude approfondie, dont l'objectif est dicté par la nature même de tout travail scientifique: repérer les constantes et les variantes, les premières l'emportant en l'espèce nettement sur les secondes.

Catherine Kerbrat-Orecchioni, professeure honoraire de l'université Lumière Lyon 2, qui a publié nombre d'articles et d'ouvrages portant notamment sur les interactions verbales, les actes de langage dans le discours ou encore les formes nominales de l'adresse en français, a travaillé sur un corpus composé des six débats organisés entre 1974 et 2012 - celui de 2017 n'est évidemment pas traité, pour des raisons évidentes de chronologie ${ }^{1}$. Il est d'emblée précisé que ce corpus a été fourni par Domitille Caillat, qui l'avait constitué pour sa thèse de doctorat ${ }^{2}$, et qu'il présente un caractère à la fois homogène et restreint qui en fait un objet d'étude idéal pour les chercheurs. 
3 Les débats de l'entre-deux-tours constituent à eux seuls un genre, à l'intersection des discours politiques (et plus spécialement électoraux) et des discours médiatiques (et plus spécialement télévisés). Il s'agit de prestations orales en interaction dans une situation de confrontation régulée. Mais cette production orale s'accompagne d'une mimogestualité qui ne peut être négligée. Elle est enfin essentiellement improvisée et co-construite, et s'adresse à un très vaste public qui va bien au-delà de l'interlocuteur et des médiateurs.

4 Contrairement à d'autres travaux ayant pris pour objet les débats d'entre-deux-tours, cette étude "s'intéresse moins à la nature des contenus échangés qu'aux "manières de faire", aux stratégies déployées par les différents participants pour parvenir à leurs fins ». Dès lors, l'approche est "essentiellement qualitative», étiquetée "discursivorhétorico-pragmatico-interactionnelle » par C. Kerbrat-Orecchioni. Après avoir rappelé l'intérêt, mais aussi les limites, de la démarche quantitative, largement mobilisée dans la littérature scientifique portant sur le discours et les débats politiques, l'auteure insiste sur les problèmes posés par la transcription.

5 Le premier chapitre s'intéresse au déroulement du débat, qui présente tous les aspects d'un rituel. C. Kerbrat-Orecchioni met en lumière les fortes similitudes observées durant toute cette période de près de quarante ans. Si quelques spécificités apparaissent, elles ne peuvent être interprétées comme la manifestation d'une claire évolution du genre. L'auteure remarque cependant une réduction de la distance interpersonnelle, qui se traduit par l'usage croissant du prénom dans les échanges entre les animateurs et, inversement, l'usage décroissant du titre de civilité Monsieur par ces derniers lorsqu'ils s'adressent aux candidats - masculins à une exception près. Mais surtout, est noté l'allongement sensible et régulier de la durée du débat, lié à la multiplication des interruptions et chevauchements, et partant l'accélération du tempo et l'augmentation de l'alternance des locuteurs.

6 Dans le deuxième chapitre, C. Kerbrat-Orecchioni aborde la question du style et des figures rhétoriques, en réservant une place particulière à l'humour. L'interactivité croissante précédemment mise au jour contribue à un abaissement du niveau de langue, notamment sur les plans phonétique et morphosyntaxique. La recherche croissante d'une plus grande proximité avec l'auditoire joue également dans le sens d'une évolution du registre vers plus de familiarité. En la matière, il est clair que le discours de Nicolas Sarkozy est emblématique de ce changement, sans le figurer à lui seul. Dans le même temps, est soulignée la relativement faible présence de l'art oratoire et de ses habituelles figures rhétoriques, souvent confinées dans les envolées finales. La spontanéité l'emporte sur l'éloquence, même si l'anaphore et la métaphore sont loin d'avoir disparu, tout comme l'hyperbole ou la question rhétorique : de fait, le propos des candidats se veut didactique, expressif et polémique, ce qui ne permet pas de négliger l'art oratoire. Si la tendance est à la sobriété stylistique, la proportion des moments «rhétorisés " semble au contraire s'accroître. Le recours à l'humour vient pimenter quelquefois ces débats qui se donnent à voir comme de plus en plus conflictuels.

7 Le troisième chapitre traite précisément de la raison d'être du débat: la mise en scène d'un affrontement qui atteint ce soir-là son paroxysme. Il apparait que la volonté de disqualifier l'adversaire l'emporte nettement sur le souci de se légitimer. Pour ce faire, chacun doit souligner son opposition à l'autre, voire l'exagérer, tandis que l'expression d'un accord est souvent ambiguë, se présentant notamment sous la forme d'une 
concession. Le désaccord, lui, est généralement dépourvu de nuances, s'exprimant par des procédés prosodiques, morphologiques, lexicaux ou stylistiques, qui rencontrent néanmoins une limite - celle qui interdit la violence verbale, sous la forme de l'injure ou de la menace notamment. Pour autant, certaines formes d'accusation d'incompétence, de sectarisme, de dureté, de mauvaise foi... ou encore l'affichage d'un rapport inégal, notamment par l'adresse, rompent avec les règles habituelles de la politesse, sans pour autant laisser la place à ce qui pourrait paraitre franchement impoli, et donc susceptible d'être contre-productif. Il faut donc polémiquer, mais sans le montrer, tout en suggérant que l'adversaire tombe dans la polémique politicienne. Là encore, C. Kerbrat-Orecchioni note des différences d'un individu à l'autre, sans vraiment constater d'évolution linéaire bien nette.

8 Enfin, le quatrième chapitre est consacré à l'autre objectif du débat, qui est la persuasion, selon les trois registres classiquement distingués en la matière (même si la réalité discursive n'est pas aussi simple): ceux du logos, de l'ethos et du pathos. Contrairement à l'idée répandue par une littérature abondante, le premier registre, celui de l'argumentation étayée, est loin de décliner. Il doit d'ailleurs largement dominer le deuxième, faute de quoi un excès d'appel aux émotions nuit à l'ethos. C'est le débat de 2007 qui a été le plus marqué par le pathos, là encore sans que cela s'inscrive dans une évolution linéaire. La place de l'ethos reste quant à elle limitée. Mais souvent, les appels à la raison, à la passion et à l'autorité sont indissociables.

9 Finalement, la continuité de ce "genre unique en son genre", que constituent les débats d'entre-deux-tours, apparaît comme une conclusion majeure de l'étude, à travers la stabilité tant des objectifs poursuivis que des moyens rhétoriques mobilisés ou des fortes contraintes qui s'imposent aux deux adversaires, quelles que soient les circonstances, mais aussi les personnalités, leur statut, leur orientation et accessoirement leur genre ${ }^{3}$ (accessoirement, puisqu'un seul des candidats de second tour a été une femme - l'enquête s'arrête avec le scrutin de 2012).

Reste à revoir et réentendre le débat du 3 mai 2017 en étant instruit de tout ce que C. Kerbrat-Orecchioni nous a appris sur ceux qui l'ont précédé. Elle terminait son ouvrage en espérant qu' " aucune circonstance particulière " ne nous en priverait. Il a bien eu lieu, mais son déroulement n'en fut pas moins " particulier ». Faudra-t-il y voir une confirmation des tendances relevées dans l'étude - assurément, il ne ressembla guère aux face-à-face Giscard/Mitterrand - ou bien un cas d'exception, l'absence de l'opposition droite/gauche et le naufrage de l'adversaire du futur élu rendant difficile son intégration dans cette belle série dont l'homogénéité était jusqu'alors si précieuse pour l'analyste ${ }^{4}$.

\section{NOTES}

1. Outre les nombreux articles et ouvrages portant sur les derniers épisodes présidentiel et législatif, la chronique en ligne «Figurez-vous » de la Société d'étude des langages du politique 
(SELP) permet de retrouver les hauts faits rhétoriques du printemps 2017 (https://selp.eu/ Figures/, consulté le 15/11/2017).

2. Domitille Caillat, Le discours rapporté dans les débats politiques télévisés: formes et fonctions des recours au discours autre. Le cas des débats de l'entre-deux-tours des présidentielles françaises (1974-2012), université Lumière Lyon 2, 2016.

3. Pour un rappel des principales caractéristiques de chacune des campagnes présidentielles de 1965 à 2012, la lecture de l'ouvrage de Gérard Courtois, chroniqueur au Monde, peut être utile : Parties de campagne. La saga des élections présidentielles, Paris, Perrin, 2017.

4. L'ouvrage dirigé par Pascal Perrineau (Le vote disruptif. Les élections présidentielle et législatives de 2017, Paris, Presses de Sciences Po, 2017, collection "Chroniques électorales») comporte un chapitre de Thierry Vedel consacré à la télévision dans la campagne électorale, et notamment au débat d'entre-deux-tours - mais il s'intéresse moins à la manière de débattre qu'aux effets du débat.

\section{AUTEURS}

\section{PAUL BACOT}

Université de Lyon, Sciences Po Lyon, Triangle (CNRS, UMR 5206) 